Ann. Sci. for., 1983, 40 (2), 165-176.

\title{
Efficacité en pépinière forestière d'un inoculum de champignon ectomycorhizien produit en fermenteur et inclus dans une matrice de polymères
}

\author{
F. LE TACON *, G. JUNG $* *$, P. MICHELOT ** et M. MUGNIER *** \\ * I.N.R.A., Station de Recherches sur les Sols forestiers, \\ Centre de Recherches forestieres de Nancy, \\ Champenoux, F 54280 Seichamps \\ ** Rhône-Poulenc Recherches, \\ Centre de Recherches de la Croix-de-Berny, \\ 182-184, aventue Aristide-Briand, F 92160 Antony Cedex
}

\section{Introduction}

Le contrôle de la mycorhization en pépinière nécessite de pouvoir produire des quantités importantes d'inoculum. Pour les champignons ectomycorhiziens, l'inoculation en pépinière d'un champignon déterminé peut se faire soit par apport de spores, soit par apport de mycélium. La première méthode a été essentiellement développée par THEODOROU (1971), THEODOROU \& BOWEN (1973) avec Rhizopogon luteolus et par MarX, Morris \& MeXal (1978) avec Pisolithus tinctorius. Cette méthode peut être utilisée industriellement par enrobage des graines, mais n'est applicable qu'aux champignons produisant beaucoup de spores germant facilement.

La seconde méthode est applicable à tous les champignons pouvant se développer en culture pure. Traditionnellement, la production d'inoculum mycélien ectomycorhizien se fait sur un mélange tourbe-vermiculite, ou sur de la vermiculite seule, saturé par une solution nutritive adéquate. Cette méthode ne présente pas de difficultés particulières lorsqu'elle est appliquée à petite échelle au laboratoire, mais est difficile à mettre en œuvre industriellement.

Nous avons cherché à produire de l'inoculum d'un champignon ectomycorhizien par une autre voie. Le mycélium est d'abord cultivé en fermenteur puis inclus dans une matrice de polymères, comme cela a été préconisé par Dommergues et al. (1977) pour les bactéries, puis par JUNG et al. (1979).

Divers types d'inoculum d'Hebeloma cylindrosportum ont été préparés suivant cette méthode, puis testés en pépinière. 


\section{Matériel et méthodes}

\subsection{Préparation des inoculums}

\subsection{Culture du microorganisme}

- Souche utilisée : Hebeloma cylindrosporum, isolé par G. BRUCHET (Université de Lyon), cultivé et entretenu sur milieu gélosé de MELin-Norkrans modifié par MARX (1969).

- Culture sur mélange tourbe-vermiculite: la souche est cultivée sur un mélange tourbe-vermiculite (1/3 de tourbe, $2 / 3$ de vermiculite) stérilisé à l'autoclave, additionné jusqu'à saturation du milieu de PACHLEWSKI, ensemencé par un fragment de culture sur milieu gélosé puis mis à incuber durant deux mois à $25^{\circ} \mathrm{C}$.

- Culture en milieu liquide: le microorganisme a été développé en fermenteur de capacité 170 à 800 litres; la séquence adoptée a été la suivante :

- culture agitée en fiole de 2 litres (ensemencée par une préculture en erlenmeyer de $300 \mathrm{ml}$ );

- culture inoculum en fermenteur de 75 à 170 litres (4 à 5 jours);

- culture productrice en fermenteur de 170 à 800 litres (2 à 3 jours).

Le milieu utilisé est un milieu à base de peptone, d'extrait de levure et de glucose (brevet FR 8104474 du 6-3-1979). Après 50 heures de culture en fermenteur de 170 litres (culture directrice) la biomasse est maximale : $4 \mathrm{~g} / \mathrm{l}$, exprimé en matière sèche à $105^{\circ} \mathrm{C}$; le mycélium se présente sous forme de microboulettes de diamètre inférieur à $1 \mathrm{~mm}$ qu'il est aisé de récupérer par simple filtration. Le gâteau obtenu est ensuite lavé 2 à 3 fois à l'eau déminéralisée.

\subsection{Inoculums avec Hebeloma cylindrosporum inclus}

Hebeloma cylindrosporum, obtenu à partir du moût de fermentation, a été inclus dans deux types de polymères :

- alginate, polymère extrait d'algue;

- polymère résultant de l'association d'un polysaccharide naturel (farine de graine de caroube) et de polysaccharide biosynthétique (gomme xanthane).

Les techniques de préparation adoptées dérivent de celles que nous avons décrites antérieurement pour l'inclusion de Rhizobium japonicum (Dommergues et al., 1977 ; JUNG, 1979), de Frankia et de champignons mycorhiziens (JUNG et al., 1981).

- Inoculums à base d'alginate (ALG): Hebeloma cylindrosporum a été inclus en s'inspirant du principe utilisé pour l'immobilisation de cellules ou d'enzymes dans de l'alginate réticulé par des ions CA++ (HaCKel et al., 1975; HACKeL, 1977 ; Kierstan \& Bucke, 1977 ; Glicksman, 1969).

- Billes d'alginate : la suspension mycélienne obtenue à partir du gâteau de filtration est additionnée d'alginate puis versée goutte à goutte dans une solution concentrée de $\mathrm{CaCl}_{2}$, ce qui permet d'obtenir des billes de 2 à $3 \mathrm{~mm}$ utilisables directement. 
- Microgranulés à base de gel d'alginate et de silice : le mélange alginate + mycélium est additionné de sulfate de calcium jusqu'à obtention d'un gel; ce gel est ensuite mélangé à 20 à 40 p. 100 de silice naturelle ou précipitée macroporeuse ou mésoporeuse; le mélange est malaxé au «Küstner», séché sous un flux d'air à $25-30^{\circ} \mathrm{C}$ jusqu'à perte de 40 à 50 p. 100 du poids, tamisé si besoin à $2 \mathrm{~mm}$ puis stocké, à température ambiante, en récipient étanche; on obtient ainsi un inoculum se présentant sous forme de «microgranulés ».

Les caractéristiques des silices les plus adaptées sont les suivantes :

- surface BET $\leqslant 200 \mathrm{~m}^{2} / \mathrm{g}$ (méthode Brunauer-Emmenteller décrite dans The Journal of the American Chemical Society, vol. 60, p. 309, 1938);

- surface CTAB $<150 \mathrm{~m}^{2} / \mathrm{g}$ (surface externe par adsorption de CTAB à pH 9, méthode JAY, JANZEN \& KRAUS dans Rubber Chemistry and Technology, 44, 1975);

- prise d'huile 100 à $400 \mathrm{ml} / 100 \mathrm{~g}$ (prise DOP).

Deux silices ont été utilisées dans cet essai :

silice $1\left(B E T=100 \mathrm{~m}^{2} / \mathrm{g}-\right.$ CTAB $=5 \mathrm{~m}^{2} / \mathrm{g}-$ DOP $\left.=350 \mathrm{ml} / 100 \mathrm{~g}\right)$ silice $2\left(B E T=150 \mathrm{~m}^{2} / \mathrm{g}-\mathrm{CTAB}=40 \mathrm{~m}^{2} / \mathrm{g}-\mathrm{DOP}=110 \mathrm{ml} / 100 \mathrm{~g}\right)$

- Inoculums à base de xanthane - graine de caroube (XG) : pour obtenir un gel à partir de xanthane, il faut lui associer un polysaccharide (1-4 galactomannane) tel que la farine de graine de caroube (Moorhouse et al., 1977), ce qui conduit à une réticulation par synergie des 2 polysaccharides.

- Microgranulés à base de gel de xanthane + caroube et de silice : le gel obtenu après inclusion du microorganisme (brevet FR 8104474, 1981) est additionné de silice et traité dans les mêmes conditions que précédemment (cf. $\$ 2.1 .2$ ): on obtient un inoculum sous forme de microgranulés.

\subsection{Inoculums à base de tourbe + vermiculite}

Deux types d'inoculums ont été préparés :

- inoculum non lavé : linoculum préparé en 2.1.1 est utilisé directement ;

- inoculum lavé : l'inoculum a été lavé abondamment à l'eau ordinaire sur tamis pendant 20 minutes, juste avant l'incorporation de l'inoculum au sol de la pépinière.

\subsection{Inoculums utilisés}

1. Billes d'alginate : $\mathbf{B}(\mathrm{ALG})$;

2. gel (xanthane + caroube) + silice $1:(X G)+S 1$;

3. gel (alginate) + silice $1:(\mathrm{ALG})+\mathrm{S} 1$;

4. gel (xanthane + caroube) + silice $2:(\mathrm{XG})+\mathrm{S} 2$;

5. mycélium lavé issu du fermenteur : (MYC);

6. mélange tourbe-vermiculite non lavé : (TV) ;

7. mélange tourbe-vermiculite lavé : (TVL). 
Les inoculums 1 à 4 ont été préparés une semaine avant la mise en place et stockés à $20-25^{\circ} \mathrm{C}$ non stérilement. L'inoculum 5 a été conservé une semaine à $+4{ }^{\circ} \mathrm{C}$. Les inoculums 6 et 7 ont été utilisés après incubation en bocal stérile et stockage pendant un mois à $+4^{\circ} \mathrm{C}$.

\subsection{Dispositif expérimental}

Le dispositif a été installé dans une pépinière de la Haute-Vienne à Peyrat-leChâteau, située à $580 \mathrm{~m}$ d'altitude (pluviosité 1200 à $1400 \mathrm{~mm}$, température moyenne annuelle $9^{\circ} \mathrm{C}$ ).

\subsection{Sol}

Le sol était originellement un sol brun ocreux humifère développé sur granite à deux micas sous une lande boisée à callune. Ce sol a été fortement fertilisé. Il est particulièrement riche en phosphore; son $\mathrm{pH}$ est de 5,5 .

\subsection{Dispositif et traitements}

Le dispositif était constitué de 2 séries de parcelles comportant respectivement 20 et 24 parcelles élémentaires de $1 \mathrm{~m}^{2}$ chacune (4 répétitions/traitement) :

la $1^{\text {re }}$ série (5 traitements) a été installée en sol désinfecté au bromure de méthyle, le 22-4-1981;

la $2^{\circ}$ série (6 traitements) a été mise en place en sol non désinfecté.

Les traitements appliqués, ainsi que les quantités correspondantes dinoculum, sont donnés dans le tableau 1.

\section{TABlead 1}

Nature et quantité des inoculums utilisés.

Type and quantity of inoculum.

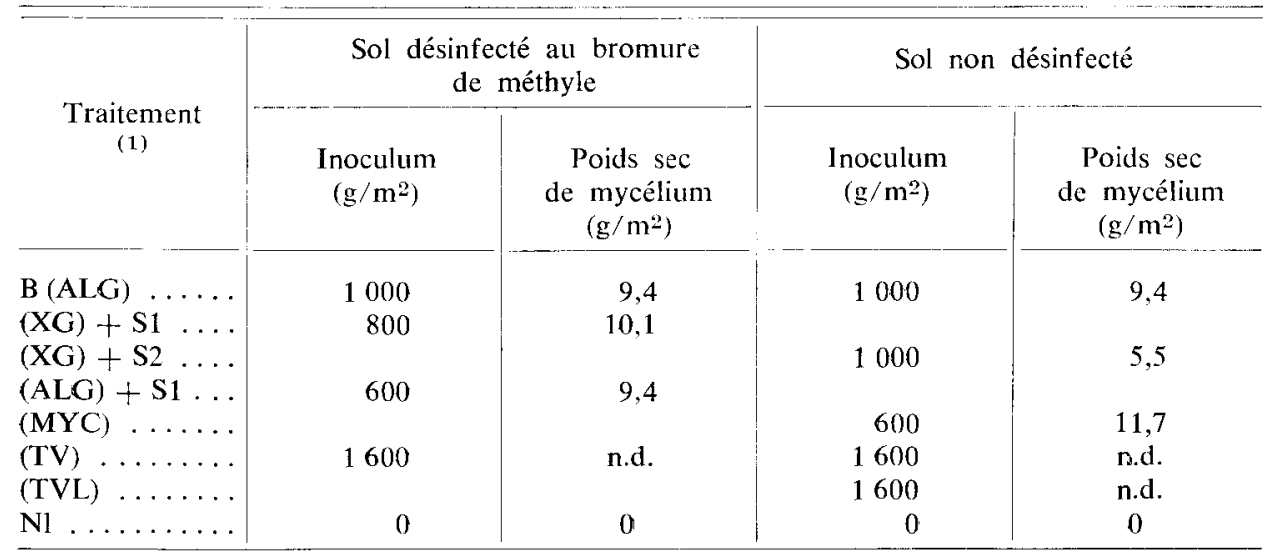

(1) Voir $\$ 2.1 .4: \mathrm{B}=$ billes; $\mathrm{ALG}=$ alginate ; $\mathrm{XG}=$ xanthane + graine de caroube ; $\mathrm{MYC}=$ mycélium $; \quad \mathrm{TV}=$ tourbe + vermiculite $; \mathrm{L}=$ lavé $; \mathrm{NI}=$ non inoculé $; \mathrm{S} 1$ et $\mathrm{S} 2=\mathrm{si}-$ lices 1 et 2 ; n.d. = non déterminé. 
- Semis : le semis a été réalisé le 6-5-1981, chaque parcelle élémentaire de $1 \mathrm{~m}^{2}$ a été ensemencée, en plein et côte à côte, avec l'épicéa commun (Picea excelsa Linn.) et le douglas (Pseudotsuga menziesii Franco).

- Inoculation : l'inoculation a été effectuée, en même temps que le semis, le 6-5-1981, par étalement de l'inoculum sur la parcelle correspondante puis enfouissement à la «griffe».

\subsection{Contrôles effectués}

6 mois après le semis (fin octobre 1981, toutes les plantules ont été arrachées avec précaution. Par parcelle élémentaire nous avons noté les caractères suivants :

- le nombre de plantules;

- la hauteur de chaque plant;

- l'intensité de la mycorhization de chaque système racinaire par $H$. cylindrosporum, appréciée par un indice allant de 0 (aucune mycorhization) à 5 (mycorhization totale des racines courtes);

- l'intensité de la mycorhization par d'autres champignons.

Les différents types de symbiotes rencontrés, outre Hebeloma cylindrosporum, étaient : Thelephora terrestris, Laccaria laccata, Boletus sp (uniquement sur douglas).

Les résultats ont été traités par analyse de variance à deux facteurs contrôlés. La ppds (plus petite différence significative) a également été déterminée.

\section{Résultats}

\subsection{Essai de mycorhization en sol désinfecté}

Cinq traitements (cf. tabl. 1) ont été appliqués sur Pseudotsuga menziesii et Picea excelsa en sol désinfecté : non inoculé $=\mathrm{NI}$; billes d'alginate $=\mathrm{B}(\mathrm{ALG})$; gel (xanthane + caroube $)+$ silice $1=(\mathrm{XG})+\mathrm{S} 1$; gel (alginate) + silice $2=(\mathrm{ALG})+\mathrm{S} 2$; tourbe + vermiculite non lavées $=\mathrm{TV}$.

\subsection{Symbiose Pseudotsuga menziesii-H. cylindrosporum}

- Mycorhization : avec les inoculums obtenus par inclusion de mycélium produit en fermenteur dans une matrice de polymères $(B(A L G)-(X G)+S 1-(A L G)+S 1)$, l'indice de mycorhization quoique assez moyen $(\sim 1,55)$ est cependant significativement supérieur à celui de l'inoculum classique tourbe + vermiculite; les résultats sont donnés dans le tableau 2.

- Levée et croissance : les parcelles inoculées, en particulier par les trois inoculums préparés à partir d'une culture en fermenteur, permettent une levée significativement supérieure à celle de la parcelle témoin avec une tendance en faveur de l'inoculum sous 
forme de billes d'alginate [B(ALG)]. Il n'y a en revanche aucune différence significative entre traitements pour la croissance en hauteur; les résultats obtenus sont donnés dans le tableau 3.

\section{TABleau 2}

Influence du type d'inoculum sur l'indice de mycorhization de Pseudotsuga menziesii en sol désinfecté (six mois après le semis).

Influence of the type of inoculum on the mycorrhization intensity of Pseudotsuga menziesii (fumigated soil - Six months after sowing).

\begin{tabular}{c|c|c|c|c|c|c}
\hline Indice de mycorhization & \multicolumn{7}{|c|}{ Inoculums } & ppds 5\% \\
\cline { 2 - 6 } & $\mathrm{NI}$ & $\mathrm{B}(\mathrm{ALG})$ & $(\mathrm{XG})+\mathrm{S1}$ & $(\mathrm{ALG})+\mathrm{S1}$ & $\mathrm{TV}$ & \\
\hline H. cylindrosporum ...... & 0 & 1,56 & 1,55 & 1,56 & 0,20 & 0,75 \\
Autres champignons ..... & 0 & 0 & 0 & 0 & 0 & - \\
\hline
\end{tabular}

(1) Indice de mycorhization allant de 0 à 5 (moyenne de tous les plants par traitement).

TABLEAU 3

Influence du type d'inoculum sur la levée et la croissance en hauteur de Pseudotsuga menziesii en sol désinfecté (6 mois après le semis).

Influence of the type of inoculum on the percentage of survival seedlings and growth of Pseudotsuga menziesii (fumigated soil - Six months after sowing).

\begin{tabular}{l|c|c|c|c|c|c}
\hline \hline & \multicolumn{5}{|c|}{ Inoculums } & ppds 5\% \\
\cline { 2 - 5 } & NI & B (ALG) & (XG) + S1 & (ALG) + S1 & TV & \\
\hline $\begin{array}{l}\text { Nombre de semis au 1/2 m² } \\
\text { Hauteur moyenne des plan- }\end{array}$ & 24,7 & 89,2 & 64,7 & 55,5 & 41,7 & 25,5 \\
tules en mm ............ & 54,7 & 59,4 & 59,2 & 57,4 & 56,0 & $\mathrm{NS}$ \\
\hline
\end{tabular}

D'une manière générale le pourcentage de levée, ainsi que la croissance en hauteur ont été très faibles en raison des mauvaises conditions climatiques du printemps qui a été froid et très pluvieux.

\subsection{Symbiose Picea excelsa - $\mathrm{H}$. cylindrosporum}

L'indice de mycorhization par Hebeloma cylindrosporum est bon avec les quatre types d'inoculum (2,0 à 3,0). L'inoculum classique sur tourbe-vermiculite (TV) est cependant significativement inférieur aux deux inoculums préparés à partir d'une culture en fermenteur (XG) + S1 et (ALG) +S1 (tabl. 4). 


\section{TABleau 4}

Influence du type d'inoculum sur l'indice de mycorhization de Picea excelsa en sol désinfecté ( 6 mois après le semis).

Influence of the type of inoculum on the mycorrhization intensity of Picea excelsa (fumigated soil - Six months after sowing).

\begin{tabular}{|c|c|c|c|c|c|c|}
\hline \multirow{2}{*}{$\begin{array}{c}\text { Indice } \\
\text { de mycorhization (1) }\end{array}$} & \multicolumn{5}{|c|}{ Inoculums } & \multirow{2}{*}{ ppds $5 \%$} \\
\hline & NiI & $\mathrm{B}(\mathrm{ALG})$ & $(X G)+S 1$ & $(A L G)+S 1$ & TV & \\
\hline H. cylindrosporum . & 0,01 & 2,49 & 2,78 & 3,03 & 2,02 & 0,50 \\
\hline Autres champignons & 0 & 0 & 0 & 0 & 0 & 一 \\
\hline
\end{tabular}

(1) Indice allant de 0 à 5 (moyenne de tous les plants par traitement).

- Levée et croissance : comme l'indique le tableau 5, le nombre de plantules par $\mathrm{m}^{2}$ ne diffère pas significativement entre les traitements; l'inoculum à base de billes d'alginate $[B(A L G)]$ assure une croissance en hauteur supérieure à celle de tous les autres traitements, bien qu'il n'ait pas le meilleur indice de mycorhization.

\section{Tableau 5}

Influence du type d'inoculum sur la levée et la croissance de Picea excelsa en sol désinfecté (6 mois après le semis).

Influence of the type of inoculum on the percentage of survival seedlings and growth of Picea excelsa (fumigated soil - Six months after sowing).

\begin{tabular}{|c|c|c|c|c|c|c|}
\hline & \multicolumn{5}{|c|}{ Inoculums } & \multirow{2}{*}{ ppds $5 \%$} \\
\hline & NI & $\mathrm{B}(\mathrm{ALG})$ & $(X G)+S 1$ & $(\mathrm{ALG})+\mathrm{S} 1$ & TV & \\
\hline Nombre de semis par $1 / 2 \mathrm{~m}^{2}$ & 18,2 & 22,2 & 17,5 & 10,7 & 15,5 & 10,5 \\
\hline $\begin{array}{l}\text { Hauteur moyenne des plan- } \\
\text { tules }(\mathrm{mm}) \ldots \ldots \ldots \ldots \ldots\end{array}$ & 41,1 & 55,6 & 46,3 & 48,1 & 45,5 & 8,1 \\
\hline
\end{tabular}

\subsection{Essai de mycorhization en sol non désinfecté}

Six traitements (cf. tabl. 1) ont été appliqués sur Pseudotsuga menziesii et Picea excelsa en sol non désinfecté : non inoculé $=\mathrm{NI}$; billes d'alginate $=\mathrm{B}(\mathrm{ALG})$; gel xanthane-caroube + silice $2=(\mathrm{XG})+\mathrm{S} 2 ;$ mycélium lavé $=(\mathrm{MYC})$; tourbe + vermiculite non lavée $=(\mathrm{TV})$; tourbe + vermiculite lavée $=$ (TVL).

\subsection{Symbiose Pseudotsuga menziesii - H. cylindrosporum}

- Mycorhization : d'une manière générale les plants sont extrêmement mal mycorhizés en sol non désinfecté (tabl. 6). L'inoculation par Hebeloma cylindrosporum est un 
échec quel que soit le type d'inoculum. Seul l'apport de mycélium non conditionné a un léger effet positif sur l'indice de mycorhization. La mycorhization par Thelephora terrestris n'est pas modifiée par l'apport d'inoculum d'Hébélome. Par contre la mycorhization par un champignon de type Boletus est améliorée lorsque l'inoculum d'Hebeloma est apporté sous forme de mélange tourbe-vermiculite non lavé. La présence des sucres et des éléments minéraux du milieu doit en être la cause.

\section{TABLeaU 6}

Influence du type d'inoculum sur l'indice de mycorhization de Pseudotsuga menziesii en sol désinfecté (6 mois après le semis).

Influence of the type of inoculum on the mycorrhization intensity of Pseudotsuga menziesii (Non fumigated soil - Six months after sowing).

\begin{tabular}{|c|c|c|c|c|c|c|c|}
\hline \multirow[b]{2}{*}{ Indice de mycorhization (1) } & \multicolumn{6}{|c|}{ Inoculums } & \multirow{2}{*}{$\begin{array}{l}\text { ppds } \\
5 \%\end{array}$} \\
\hline & NI & B (ALG) & $\begin{array}{l}(X G) \\
+S 2\end{array}$ & MYC & TV & TVL & \\
\hline Hebeloma cylindrosporum & 0,09 & 0,09 & 0,19 & 0,27 & 0,005 & 0,06 & 0,20 \\
\hline Thelephora terrestris . . . . . & 0,015 & 0 & 0,017 & 0,027 & 0,06 & 0,02 & NS \\
\hline Boletus sp .............. & 0,12 & 0,19 & 0,03 & 0,10 & 0,51 & 0,34 & $0 ; 39$ \\
\hline Laccaria laccata .. & 0 & 0 & 0 & 0 & 0 & 0 & NS \\
\hline
\end{tabular}

(1) Indice allant de 1 à 5 (moyenne de tous les plants par traitement).

\section{TABLEAU 7}

Influence du type d'inoculum sur la levée et la croissance en hauteur de Pseudotsuga menziesii en sol désinfecté (6 mois après le semis).

Influence of the type of inoculum on the percentage of survival seedlings and growth of Pseudotsuga menziesii (Non fumigated soil - Six months after sowing).

\begin{tabular}{|c|c|c|c|c|c|c|c|}
\hline & \multicolumn{6}{|c|}{ Inoculums } & \multirow{2}{*}{$\begin{array}{l}\text { ppds } \\
5 \%\end{array}$} \\
\hline & NI & $\mathrm{B}(\mathrm{ALG})$ & $\begin{array}{l}\text { (XG) } \\
+\mathrm{S} 2\end{array}$ & MYC & TV & TVL & \\
\hline Nombre de semis par $1 / 2 \mathrm{~m}^{2}$ & 34,5 & 23,0 & 31,5 & 29,2 & 49,7 & 30,2 & 18,0 \\
\hline Jauteur moyenne en $\mathrm{mm}$ & 55,4 & 55,3 & 55,6 & 57,5 & 63,0 & 58,5 & 5,3 \\
\hline
\end{tabular}

- Levée et croissance : Il ressort du tableau 7, que par rapport au sol désinfecté la hauteur et le nombre de plantules de douglas ne sont pas significativement différents six mois après l'inoculation. On notera cependant que l'inoculation en sol non désinfecté n'améliore pas le nombre de semis, sauf pour l'inoculum tourbe-vermiculite non lavé, alors que cette amélioration était très nette en sol désinfecté (cf. tabl. 3). 
Les différents types d'inoculums n'ont pas d'effet sur la croissance en hauteur, sauf pour l'inoculum tourbe-vermiculite non lavé qui a un léger effet. Ce léger effet de l'inoculum non lavé tourbe-vermiculite sur le nombre de semis et la croissance, peut être attribué, soit directement à l'effet sur l'arbre des éléments minéraux ou organiques du milieu, soit à son effet indirect sur la mycorhization par lc champignon de type Bolet.

\subsection{Symbiose Picea excelsa - H. cylindrosporum}

- Mycorhization : comme pour le douglas, l'épicéa est très mal mycorhizé en sol non désinfecté (tabl. 8).

L'inoculation par Hebeloma cylindrosporum est également un échec quel que soit le type d'inoculum. On notera qu'Hebeloma cylindrosporum ou un autre hébélome est présent dans le témoin, mais l'indice de mycorhization est très faible $(0,02$ à 0,38$)$.

\section{TABLEAU 8}

Iniluence du type d'inoculum sur l'indice de mycorhization de Picea excelsa en sol non désinfecté ( 6 mois après le semis).

Influence of the type of inoculum on the mycorrhization intensity of Picea excelsa (Non fumigated soil - Six months after sowing).

\begin{tabular}{|c|c|c|c|c|c|c|c|}
\hline \multirow[b]{2}{*}{ Indice de mycorhization (1) } & \multicolumn{6}{|c|}{ Inoculums } & \multirow{2}{*}{$\begin{array}{l}\text { ppds } \\
5 \%\end{array}$} \\
\hline & NI & B (ALG) & $\begin{array}{l}(\mathrm{XG}) \\
+\mathrm{S} 2\end{array}$ & MYC & TV & TVL & \\
\hline Hebeloma cylindrosporum & 0,38 & 0,16 & 0,32 & 0,16 & 0,06 & 0,02 & NS \\
\hline Thelephora terrestris & 0,37 & 0 & 0,30 & 0,31 & 1,27 & 1,04 & 0,78 \\
\hline Laccaria laccata .. & 0,12 & 0,06 & 0 & 0,12 & 0 & 0 & NS \\
\hline Boletus sp ....... & 0 & 0 & 0 & 0 & 0 & 0 & 一 \\
\hline
\end{tabular}

(1) Indice allant de 1 à 5 (moyenne de tous les plants par traitement).

\section{TABLeaU 9}

Influence du type d'inoculum sur la levée et la croissance en hauteur de Picea excelsa en sol non désinfecté ( 6 mois après le semis).

Influence of the type of inoculum on the percentage of survival seedlings and growth of Picea excelsa (Non fumigated soil - Six months after sowing).

\begin{tabular}{|c|c|c|c|c|c|c|c|}
\hline & \multicolumn{6}{|c|}{ Inoculums } & \multirow{2}{*}{$\begin{array}{l}\text { ppds } \\
5 \%\end{array}$} \\
\hline & NI & $B(A L G)$ & $\begin{array}{l}(\mathrm{XG}) \\
+\mathrm{S} 2\end{array}$ & MYC & TV & TVL & \\
\hline Nombre de semis par $1 / 2 \mathrm{~m}^{2}$ & 3,5 & 11 & 6,7 & 8,2 & 31,7 & 23,2 & 16 \\
\hline Hauteur moyenne en $\mathrm{mm}$ & 33,6 & 36,6 & 44,5 & 42,7 & 51,2 & 49,4 & NS \\
\hline
\end{tabular}


Au contraire de ce que nous avons observé pour l'épicéa, la mycorhization par Thelephora terrestris est augmentée par l'apport d'inoculum d'hébélome sous forme de mélange tourbe-vermiculite non lavé, toujours probablement en raison de la présence de sucres et d'éléments minéraux dans le milieu. Il n'y a par contre pas d'effet sur la mycorhization par Laccaria laccata. On notera que les mycorhizes de type Boletus, que nous rencontrons sur douglas, ne s'observent jamais sur épicéa.

- Levée et croissance : il n'y a aucun effet du type d'inoculum sur la croissance en hauteur de l'épicéa (tabl. 9). Par contre, l'inoculum tourbe-vermiculite, qu'il soit lavé ou non lavé, améliore significativement le nombre de semis par $\mathrm{m}^{2}$.

\section{Discussion et conclusions}

En 1981, les conditions climatiques ont été extrêmement défavorables à la pépinière de Peyrat-le-Château (très basses températures en mai avec excès de pluviosité, basses températures pendant la saison de végétation). Il s`en est suivi une très mauvaise germination, une germination très échelonnée daris le temps pour le douglas ( 3 mois) et une mauvaise croissance générale, aussi bien pour l'épicéa que pour le douglas.

Il n'est donc pas étonnant que les différents traitements n'aient cu qu'une faible influence sur la croissance en hauteur des plants. Le but essentiel de ces essais était de comparer l'efficacité de différents types d'inoculums d'Hebeloma cylindrosporum sur le développement de la mycorhization.

En sol non désinfecté, il apparaît impossible d'inoculer de manière satisfaisante Hebeloma cylindrosporum, quel que soit le type d'inoculum (indice de mycorhization $\leqslant 0,30$ - inoculum classique lavé ou non lavé, inoculum produit en fermenteur et inclus ou non dans des réseaux de polymères). Ce résultat est assez surprenant, car il ne semble pas qu'il $\mathrm{y}$ ait un problème de compétition entre champignon introduit et champignons préexistants dans le sol. En effet, en sol non désinfecté la mycorhization naturelle est extrêmement faible : très faible mycorhization naturelle par Thelephora terrestris et Laccaria laccata pour l'épicéa (indice de mycorhization $=0,37$ et 0,12 respectivement) et pour le douglas par Thelephora terrestris et un champignon non déterminé de type Boletus (mycorhizes blanches, manteau feutré, nombreux rhizomorphes blancs - indice de mycorhization $=0,015$ et 0,12 respectivement).

Il semble donc que la cause de l'échec de l'inoculation par Hebeloma cylindrosporum en sol non désinfecté soit plutôt dû à la compétition avec la microflore totale du sol, qu'à une concurrence avec les autres champignons ectomycorhiziens présents naturellement dans le sol de la pépinière.

On notera que l'inoculum d'Hebeloma cylindrosporum, apporté sous forme de mélange non lavé de tourbe et de vermiculite, a un effet favorable sur le développement de la mycorhization par d'autres champignons ectomycorhiziens présents naturellement dans le sol. Cet effet est net pour les mycorhizes de Thelephora terrestris dans le cas de l'épicéa (indice de mycorhization $=1,27$ contre 0,37 pour NI) et pour les mycorhizes de type Bolet dans le cas du douglas (indice de mycorhization $=0,51$ contre $0,12 \mathrm{NI}$ ). 
En sol désinfecté au bromure de méthyle l'inoculation d'Hebeloma cylindrosporum s'effectue de manière très satisfaisante et de façon sensiblement identique en particulier avec les 3 inoculums préparés à partir de mycélium produit en fermenteur et inclus dans des polymères (billes d'alginate, mélange gel-xanthane-farine de caroube + silice macroporeuse, gel alginate + silice macroporeuse).

Pour le douglas, la mycorhization est assez moyenne (indice de mycorhization $=1,56)$ avec les polymères et extrêmement mauvaise avec l'inoculum lavé tourbevermiculite (indice de mycorhization $=0,20$ ).

Pour l'épicéa, la mycorhization est excellente avec l'inoculum inclus dans les polymères (indice de mycorhization $=2,49$ à 3,03 ) et un peu moins bonne avec l'inoculum non lavé tourbe-vermiculite. Cette différence entre les deux espèces est à mettre en parallèle avec les difficultés de germination. Pour l'épicéa la germination a été très mauvaise (peu de graines ont germé), mais relativement rapide (levée 3 semaines après le semis). Le mycélium des 4 types d'inoculum, y compris celui de l'inoculum tourbevermiculite, a survécu jusqu'à ce que les racines deviennent réceptives à la mycorhization.

Pour le douglas la germination a été très échelonnée dans le temps, la plupart des graines n'ont germé qu'après 3 mois et les racines réceptives ne sont apparues qu'après 4 mois.

Le mycélium inclus dans les polymères a relativement bien résisté, restant suffisamment infectieux pour induire la mycorhization 4 mois après linoculation. Par contre, le mycélium de l'inoculum classique tourbe-vermiculite n'a probablement pas pu résister durant 4 mois, ce qui pourrait expliquer sa très faible efficacité sur douglas, du moins dans les conditions de 1981 .

Il apparaît donc que cette méthode de production d'inoculum de champignon ectomycorhizien en fermenteur avec inclusion dans des gels de polymères s'avère très efficace et plus efficace, au moins dans les conditions de notre essai, que l'inoculum classique produit sur mélange tourbe-vermiculite.

Il reste à contrôler l'efficacité de cette méthode pour d'autres champignons et d'autres essences forestières et à préciser les possibilités de conservation de ce type d'inoculum.

Reçu pour publication le 30 juin 1982.

\section{Summary}

\section{Efficiency in a forest nursery of an inoculum of an ectomycorrhizal fungus} produced in a fermentor and entrapped in polymetric gels

Pure culture inocula of ectomycorrhizal fungi are usually produced on a vermiculite peat mixture. They can also be prepared by cultivating the fungus in a fermentor and entrapping it in a polymeric gel as proposed by Dommergues et al. (1979) for bacteria and JUNG et al. (1981) for different microorganisms. We have prepared different types of inoculum of Hebeloma cylindrosporum as follows :

- non washed peat vermiculite inoculum;

- washed peat vermiculite inoculum;

- pellets of mycelium cultivated in a fermentor of 800 liters;

- pellets of mycelium cultivated in a fermentor and included in an alginate gel ; 
- pellets of mycelium cultivated in a fermentor, included in an alginate gel and mixed with macropourous silica ;

- pellets of mycelium cultivated in a fermentor, included in a gel of xanthane and 1-4 galactomannan (caroube gel) and mixed with macropourous silica.

These different types of inoculum have been tested with spruce (Picea exelsa) and douglas fir (Pseudotsuga douglasii) in fumigated and non fumigated nursery soil.

In non fumigated soil it is impossible to obtain a good mycorrhizal development by Hebeloma cylindrosporum with any of the above types of inoculum.

In fumigated soil we have obtained a good mycorrhizal development of the two species with Hebeloma cylindrosporum.

The mycelium entrapped in the three types of polymers (alginate, alginate and macropourous silica, xanthane, galactomannan and macropourous silica) is a better inoculum than the non entrapped mycelium or the mycelium produced on peat and vermiculite.

This method of producing mycelium of an ectomycorrhizal fungus in fermentor and entrapping it in a polymeric gel seems to be a suitable way for producing large quantities of commercial inoculum.

\section{Références bibliographiques}

Dommergues Y., Hoang G. Diem, Divies Ch. Brevet US 4155737 du 22-5-1979 : Microbiological process for controlling the productivity of cultivated plants.

JUNG G. Brevet FR 7908597 du 5-4-1979 : Procédé d'inclusion de microorganismes dans une matrice polymère et produit ainsi obtenu.

Glicksman A.H., 1969. Gum technology in the food industry. Academic Press, New York, 245-246.

Hackel U., Klein J., Megnet R., Wagner F., 1975. Immobilization of microbial cells in polymeric matrices. Eur. J. appl. Microbiol., 1, 291-293.

HACKel U., 1977. Polymereinschluss von Mikroorganismen zu Aufbau und Reaktivität von Biokatalysatoren. Thesis, Naturwissenschaftlichen Fakultät der Technischen Universität Carolo-Wilhelmina zu Braunschweig, 108 p.

Kierstan M., Bucke C., 1977. The immobilization of microbial cells, subcellular organelles and enzymes in calcium alginate gels. Biotech, Bioengin., 14, 387-397.

Le Tacon F., Valdenaire J.M., 1980. La mycorhization contrôlée en pépinière. Premiers résultats obtenus à la pépinière du Fonds forestier national de Peyrat-le-Château (Haute-Vienne) sur épicéa (Picea excelsa) et douglas (Pseudotsuga douglasii). Rev. for. fr., $X X X I I, 3,281-285$.

Marx D.H., Morris W.G., Mexal J.G., 1978. Growth and ectomycorrhizal development of loblolly pine seedlings in fumigated and non fumigated nursery soil infested with different fungal symbionts. Forest Science, 24 (2), 193-203.

Marx D.H., 1980. Ectomycorrhizal fungus inoculations : a tool for improving forestation practice. In : Tropical mycorrhiza research (P. Mikola ed.), 13-71, Oxford University Press, Oxford.

Moorhouse R., Walkinshaw M.D., ArnotT S., 1977. Xanthan gum-molecul conformation and interactions. In : Extracellular microbial polysaccharid A.C.S. Symposium (P.A. Sandford and A. Laskin eds), Washington D.C. 45, 90-102.

Theodorou C., 1971. Introduction of mycorrhizal fungi into soil by spores inoculation of seeds. Aust. For., 35, 23-26.

Theodorou C., Bowen G.D., 1973. Inoculation of seeds and soil with basidiospores of mycorrhizal fungi. Soil Biol. Biochem., 5 (6), 765-771.

Trappe J.M., 1977. Selection of fungi for ectomycorrhizal inoculation in nurseries. Annu. Rev. Phytopathol., 15, 203-222. 